\title{
Assessment of Cardiovascular Risk Factors and Their Interactions in the Risk of Coronary Heart Disease in Patients with Type 2 Diabetes with Different Weight Levels, 2013-2018
}

\author{
Chenchen Wang ${ }^{1,2}$ \\ Demei $\mathrm{Ye}^{\prime}$ \\ Zuoling $\mathrm{Xie}^{1,2}$ \\ Xi Huang' \\ Zheng Wang ${ }^{2}$ \\ Haiyan Shangguan ${ }^{2,3}$ \\ Wenwen $\mathrm{Zhu}^{2}$ \\ Shaohua Wang' \\ 'Department of Endocrinology, The \\ Affiliated ZhongDa Hospital of Southeast \\ University, Nanjing, 210009, People's \\ Republic of China; ${ }^{2}$ School of Medicine, \\ Southeast University, Nanjing, 210009 , \\ People's Republic of China; ${ }^{3}$ Nanjing \\ Central Hospital, Nanjing, 210008, \\ People's Republic of China
}

Correspondence: Shaohua Wang Department of Endocrinology, The Affiliated ZhongDa Hospital of Southeast University, Nanjing, 210009, People's Republic of China

Tel +862583262810

Fax +862583285I32

Email gyjwsh@I26.com
Purpose: In addition to hyperglycemia and hyperlipidemia, obesity and hypertension are important cardiovascular risk factors for coronary heart disease (CHD) in patients with type 2 diabetes mellitus (T2DM). This study aims to explore the interaction of these risk factors. Patients and Methods: Data of hospitalized patients with T2DM from 2013 to 2018 were collected. A multivariate logistic regression model was established. Patients with normal weight and blood pressure were recruited as controls. The interaction on the risk of CHD was evaluated by relative excess risk due to interaction (RERI).

Results: Among the 30,693 patients with T2DM, 7202 (23.5\%) had CHD. In the low weight group, the prevalence of CHD in patients with hypertension was nearly four times higher than that in patients without hypertension $(42.7 \%$ vs $11.3 \%, \mathrm{P}<0.01)$. The OR value of hypertension alone on CHD increased from 1.29 (95\% CI 1.06-1.56) in the body mass index (BMI) $30.0-34.9 \mathrm{~kg} / \mathrm{m}^{2}$ group to 1.35 (95\% CI $\left.1.11-1.62\right)$ in the $\mathrm{BMI} \leq 18.5 \mathrm{~kg} / \mathrm{m}^{2}$ group. Additive interaction was observed between hypertension and BMI in CHD risk, especially in the low weight group (RERI:2.2, 95\% CI 0.9-3.5). BMI and CHD risk showed a smile curve relationship. The attributive proportion in the low weight group was higher than that in the severe obesity group, that is, 0.52 (95\% CI $0.35-0.69)$ vs $0.18(95 \%$ CI -0.59 to 0.95$)$.

Conclusion: Maintaining normal weight and avoiding low weight are particularly important for patients with co-occurring diabetes and hypertension to prevent the risk of CHD.

Keywords: type 2 diabetes, cardiovascular, hypertension, body mass index, interaction

\section{Introduction}

In 1999, the American Heart Association (AHA) identified diabetes as a cardiovascular disease and has officially listed this condition as a major risk factor for coronary heart disease (CHD), along with dyslipidemia, hypertension, and smoking. The New England Journal of Medicine published a 7-year Finnish study, ${ }^{1}$ which suggested that patients diagnosed with diabetes are expected to face the same risk of CHD as those with a history of myocardial infarction. In 2001, the National Cholesterol Education Program Adult Treatment Panel III listed diabetes as an equal-risk disease for CHD, that is, patients with diabetes without CHD have the same risk of developing CHD as non-diabetics with a history of CHD. ${ }^{2}$ This investigation further revealed that ${ }^{3}$ the risk of cardiovascular disease in patients with diabetes was two to four times higher than in those without diabetes. In 
patients with diabetes but no history of myocardial infarction, the risk of having a myocardial infarction in the next $8-10$ years is $20 \%$, which is equal to the risk of having a subsequent myocardial infarction in patients who have already had a myocardial infarction. Patients with diabetes who have already suffered myocardial infarction have a greater than $40 \%$ risk of future myocardial infarction, and those with cardiovascular disease have worse prognosis than non-diabetics. ${ }^{4,5}$

Obesity, hypertension, hyperlipidemia, smoking, and other cardiovascular risk factors are common clustered in patients with diabetes. ${ }^{6}$ Therefore, prevention and treatment strategies for CHD have been proposed based on metabolic risk factors. The 2020 ADA Guidelines $^{7}$ suggested that for patients with diabetes and hypertension, blood pressure control goals should be set according to cardiovascular disease risk stratification: blood pressure should be controlled at $<130 / 80 \mathrm{mmHg}$ when the risk of cardiovascular disease is high and should be within $<140$ / $90 \mathrm{mmHg}$ when the risk is low. Most of previous studies independently analyzed risk factors. However, if only one index is emphasized and enhanced to reach the target, then the other risks may be increased. For example, intensive hypoglycemia had no significant effect on the occurrence of microvascular complications and major cardiovascular events, ${ }^{8,9}$ but increases patient mortality. ${ }^{9}$ Cardiovascular disease risk depends on the severity of a particular risk factor and on the clustering of risk factors in the individual. ${ }^{10}$ Interactions occur among these clustering risk factors. Studies based on interactions between metabolic risk factors revealed an additive interaction between BMI and gestational hypertension for type 2 diabetes risk, and $36 \%$ of the risk can be attributed to this interaction. ${ }^{11}$ An additive interaction was also observed between BMI and gestational hypertension history for chronic hypertension risk. ${ }^{12}$ The interaction between BMI and hypertension could be explained that BMI and hypertension are causally and closely interrelated, and they have the same pathophysiological basis, for example, insulin resistance, sympathetic dysfunction. ${ }^{13}$ However, most studies on CHD interactions have focused on gene-gene ${ }^{14}$ or environmental-induced epigenetic changes. ${ }^{15}$ The interaction between GSTM1 deletion genotype and smoking status seriously influences the CHD risk. ${ }^{16}$ Work stress and social stress have a synergistic effect ${ }^{17}$ on increasing the risk of $\mathrm{CHD}$. Only a few studies have examined the value of the interaction of metabolic factors in the prevention of CHD. No research has been conducted to determine whether maintaining a reasonable body weight in patients with cooccurring diabetes and hypertension has the same benefit in low weight and obese patients in preventing CHD.

To assess the cardiovascular risk factors and their interactions in the risk of coronary heart disease in patients with type 2 diabetes, we collected medical history, physical measurements, blood-glucose, HbAlc, blood lipid, etc. in a large sample of 30,693 patients hospitalized between 2013 and 2018. This research aimed to identify high-risk groups for the early intervention of risk factors and to adopt individualized CHD prevention strategies for different populations.

\section{Patients and Methods Study Population}

The study population included patients hospitalized in the ZhongDa Hospital affiliated to Southeast University from July 2013 to December 2018. To avoid selection bias, we included all patients consecutively, then a total of 66,536 cases with diabetes were enrolled according to the primary discharge diagnosis. After excluding 5411 cases of type 1 diabetes, specific type diabetes, gestational diabetes, and diabetes not clearly classified, 61,125 cases with T2DM remained. Only data from the first hospitalization $(n=31,112)$ were used for patients with multiple hospitalizations during this period. After excluding 419 patients with severely missing data, a total of 30,693 inpatients (16,709 men and 13,984 women) were included eventually. A flowchart of the study procedure is shown in Figure 1. This study was approved by the Research Ethics Committee of the Affiliated ZhongDa Hospital of Southeast University (Approved No. of ethic committee: 2020ZDSYLL028-P01).

\section{Data Collection}

We collected detailed data on each patient, including their demographic variables: gender, age, and ethnicity; Medical history: diabetes mellitus, coronary heart disease, hypertension; Smoking and drinking were identified according to the medical records of the patients; Physical measurements: weight, height, body mass index (BMI); Essential medication information: insulin, metformin, antiplatelet aggregation, and statins; Venous blood samples were collected in the morning after a nighttime fast of at least 8 hours and all blood specimens were sent to the laboratory for examination of fasting blood-glucose (FBG), HbA1c, total-cholesterol (T-CHOL), triglyceride, 


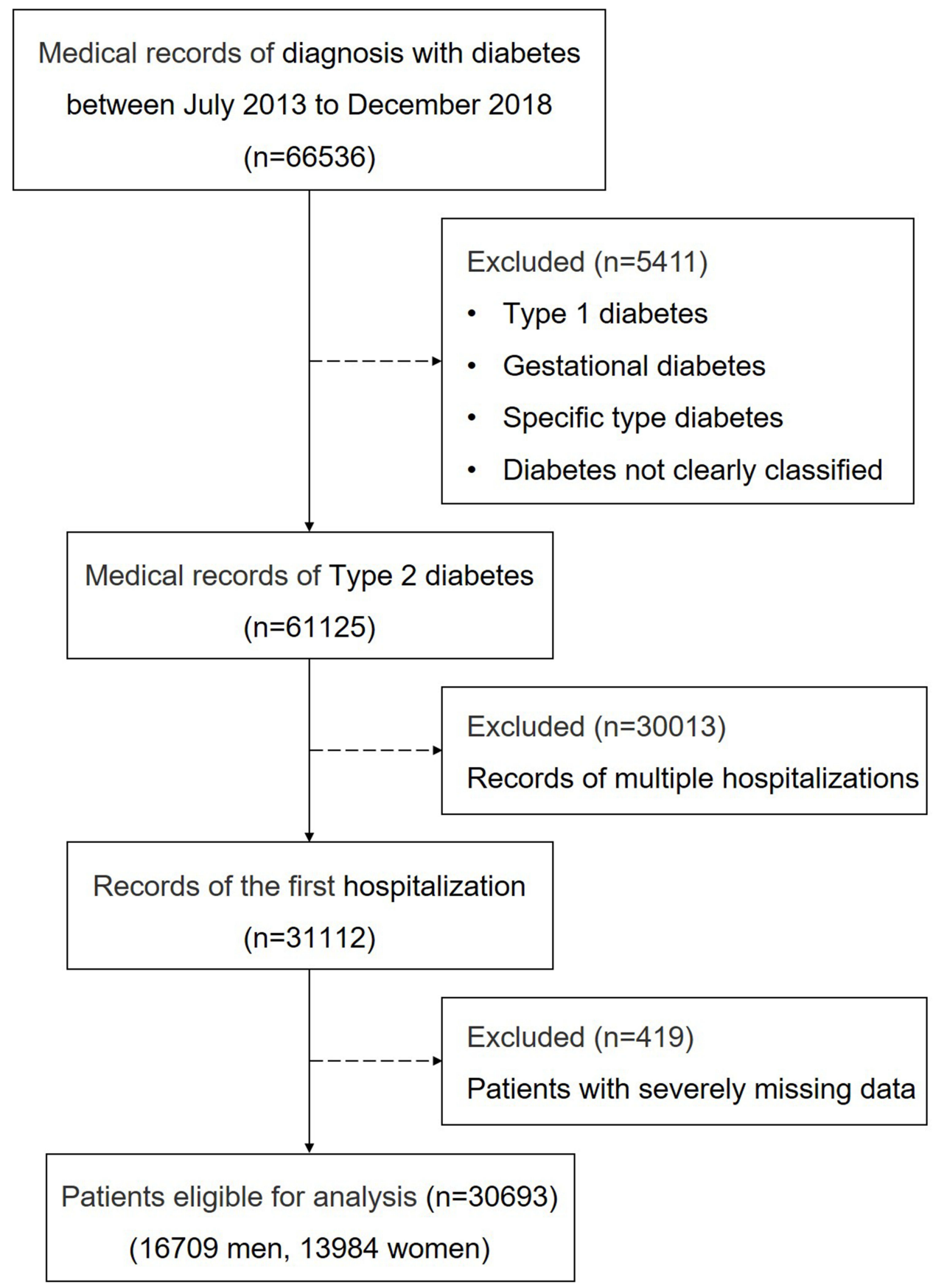

Figure I Flowchart of study participants.

high-density lipoprotein cholesterol (HDL-C), low-density lipoprotein cholesterol (LDL-C), apolipoprotein-A1, apolipoprotein-B, lipoprotein-a (Lpa), blood urea nitrogen (BUN), serum creatinine (SCr), serum uric acid (SUA), prothrombin time (PT), activated partial thromboplastin time (APTT), thrombin time (TT), fibrinogen, antithrombin-III, fibrinogen degradation product (FDP) and D dimer. The Laboratory center of Zhongda Hospital Affiliated to Southeast University implements internal and external quality management procedures directed by the Chinese Laboratory Quality Control. All blood samples were analyzed by professional clinical laboratory medical staff of Zhongda Hospital.

\section{Definition of Variables}

We extracted the principal and other diagnoses of discharge diagnosis for each case, which were coded with the International Classification of Diseases (ICD)-10. All 
diseases were identified by the corresponding code, including diabetes (ICD-10 codes E10-14), coronary heart disease (ICD-10 codes I20-I25), and hypertension (ICD-10 codes I10). Hypoglycemia was defined as having a blood glucose level of $\leq 3.9 \mathrm{mmol} / \mathrm{L}$ in diabetics.

We calculated BMI as the weight in kilograms divided by the square of the height in meters and parameterized BMI into five categories: low weight $\left(<18.5 \mathrm{~kg} / \mathrm{m}^{2}\right)$, normal weight (18.5 to $\left.<25.0 \mathrm{~kg} / \mathrm{m}^{2}\right)$, over weight $(25.0$ to $<30.0 \mathrm{~kg} / \mathrm{m}^{2}$ ), mild obesity $\left(30.0\right.$ to $<35.0 \mathrm{~kg} / \mathrm{m}^{2}$ ), and severe obesity $\left(\geq 35.0 \mathrm{~kg} / \mathrm{m}^{2}\right)$.

\section{Statistical Analysis}

About $8.7 \%$ data were missing. In order to reduce the impact of missing data, we used multiple imputation with 5 imputations, and the final estimation results were calculated by the multivariate model. All analyses were performed on the pooled data sets, which were combined into complete data according to Rubin standard rules.

We used binary non-conditional logistic regression models to estimate odds ratio (OR) for the association between BMI and coronary heart disease by history of hypertension. These models included BMI, hypertension, and their interaction terms. In the first step of adjustment, we adjusted for sex, age, and diabetes duration. Our main model additionally included smoking, drinking, use of antiplatelet, statin, insulin, and metformin, FBG, HbAlc, hypoglycemia, total cholesterol, LDL-C, triglyceride, Lpa, $\mathrm{SCr}$, and SUA.

By comparing the individual and combined effects of BMI and hypertension in CHD incidence, we calculated the additive interaction between BMI and hypertension for the risk of CHD. We used patients with BMI 18.5 to $<25.0 \mathrm{~kg} / \mathrm{m}^{2}$ and without hypertension as the reference group in multivariate regression analysis. To explore the additive interaction between BMI and hypertension, relative excess risk due to interaction (RERI) ( $>0$ supports additive interaction), attributable proportion (AP) due to interaction, and synergy index (SI) were calculated in each categories of BMI using the Excel spreadsheet prepared by Andersson. ${ }^{18}$ We also analyzed the multiplicative interaction using logistic regression models.

Numeric variables with or without normal distribution were presented as mean \pm standard deviation (SD), median (interquartile range), respectively. Qualitative variables were shown as the number (percentage). Differences between groups were compared using 1-way analysis of variance or Kruskal-Wallis tests for continuous data, and then pairwise comparisons of overall significance were performed using post hoc tests. Chi-squared $\left(\chi^{2}\right)$ test were used for comparison of qualitative variables. SPSS 21 (SPSS Inc., Chicago, IL, USA) and Microsoft Excel 2019 was used for analysis.

\section{Results}

\section{Demographic and Clinical Characteristics}

A total of 30,693 hospitalized patients with type 2 diabetes were enrolled in this study. Table 1 shows the demographic and clinical characteristics of each group after grouping according to BMI. Compared with the normal weight group, the patients in the low weight group were older and had longer duration of diabetes, lower rates of hypertension and smoking, and higher levels of HbAlc, hypoglycemia, and Lpa. The overweight and obese groups were younger and had shorter duration of diabetes, higher rates of hypertension and smoking, and lower levels of HbA1c, hypoglycemia, and Lpa ( ${ }^{\mathrm{a}}$ represents $\mathrm{P}<0.05$ ).

\section{Relationship Between Cardiovascular Risk Factors and Coronary Heart Disease}

Figure 2 and Table 2 show the relationship between body weight and CHD prevalence after the addition of four cardiovascular risk factors (hypertensive, HbA1c, Lpa, and smoking). A panel shows that among patients without hypertension, the highest prevalence of CHD was in the overweight group (13.0\%), and the lowest were in the low weight $(11.3 \%)$ and severe obesity $(9.4 \%)$ groups at both ends of BMI. For those with hypertension complication, the prevalence of CHD was the lowest in the overweight group (27.8\%) and the highest in those with low weight (42.7\%) and severe obesity (33.7\%) groups at both ends of BMI. In the low weight group, the prevalence of CHD in patients with hypertension was nearly four times higher than that in patients without hypertension $(42.7 \%$ vs $11.3 \%, \mathrm{P}<0.01)$. This finding indicates that hypertension was the most influential cardiovascular risk factor for the prevalence of CHD in different BMI populations. Other cardiovascular risk factors (HbA1c $\geq 7 \%, \mathrm{Lpa}>300 \mathrm{mg} / \mathrm{L}$, smoking) were not as significant as hypertension (Figure $2 \mathrm{~B}-\mathrm{D}$ ).

Table 2 also shows the risk of CHD in different BMI groups stratified according to the presence or absence of hypertension. Among the people with hypertension, BMI and CHD risk presented a smile curve, with the highest risk found in the low weight (OR 1.78, 95\% CI 1.43-2.23) and severe obesity (OR 1.72, 95\% CI 1.24-2.39) groups at 
Table I Descriptive Characteristics of the Study Sample at Classified by BMI

\begin{tabular}{|c|c|c|c|c|c|c|}
\hline & $\begin{array}{l}\text { Low Weight } \\
\left(<18.5 \mathrm{~kg} / \mathrm{m}^{2}\right) \\
n=967\end{array}$ & $\begin{array}{l}\text { Normal Weight } \\
\left(18.5-24.9 \mathrm{~kg} / \mathrm{m}^{2}\right) \\
n=\mid 4,443\end{array}$ & $\begin{array}{l}\text { Overweight } \\
\left(25.0-29.9 \mathrm{~kg} / \mathrm{m}^{2}\right) \\
n=\mid 2,204\end{array}$ & $\begin{array}{l}\text { Mild Obesity } \\
\left(30.0-34.9 \mathrm{~kg} / \mathrm{m}^{2}\right) \\
\mathrm{n}=2769\end{array}$ & $\begin{array}{l}\text { Severe Obesity } \\
\left(\geq 35.0 \mathrm{~kg} / \mathrm{m}^{2}\right) \\
n=310\end{array}$ & $P$ value \\
\hline Female, n (\%) & $488(50.5)^{a}$ & $6493(45)$ & $545 I(44.7)$ & $1374(49.6)^{a}$ & $178(57.4)^{\mathrm{a}}$ & $<0.001$ \\
\hline Age (year) & $71.5 \pm 12.5^{\mathrm{a}}$ & $68.4 \pm 12.4$ & $66.8 \pm 12.9^{\mathrm{a}}$ & $65.0 \pm 14.7^{\mathrm{a}}$ & $61.9 \pm 17.1^{\mathrm{a}}$ & $<0.001$ \\
\hline Diabetes duration (y) & $8.5 \pm 3.7^{\mathrm{a}}$ & $8.0 \pm 3.4$ & $7.8 \pm 3.3^{\mathrm{a}}$ & $7.7 \pm 3.8^{\mathrm{a}}$ & $6.7 \pm 3.8^{\mathrm{a}}$ & $<0.001$ \\
\hline Hypertension, n (\%) & $506(52.3)^{\mathrm{a}}$ & $8784(60.8)$ & $8581(70.3)^{\mathrm{a}}$ & $2170(78.4)^{\mathrm{a}}$ & $246(79.4)^{a}$ & $<0.001$ \\
\hline CHD, n (\%) & $268(27.7)^{\mathrm{a}}$ & $3235(22.4)$ & $2852(23.4)$ & $758(27.4)^{\mathrm{a}}$ & $89(28.7)$ & $<0.001$ \\
\hline Smoking, n (\%) & $162(16.9)^{\mathrm{a}}$ & $3017(21.2)$ & $283 I(23.5)^{\mathrm{a}}$ & $716(26.2)^{\mathrm{a}}$ & $80(26.6)$ & $<0.001$ \\
\hline Drinking, n (\%) & $69(7.2)^{a}$ & $1656(11.6)$ & $1656(13.7)^{\mathrm{a}}$ & $415(15.2)^{\mathrm{a}}$ & $44(14.6)$ & $<0.001$ \\
\hline BMI $\left(\mathrm{kg} / \mathrm{m}^{2}\right)$ & $17.1 \pm 1.2^{\mathrm{a}}$ & $22.5 \pm 1.7$ & $27.0 \pm 1.4^{\mathrm{a}}$ & $31.6 \pm 1.3^{\mathrm{a}}$ & $37.4 \pm 3.6^{\mathrm{a}}$ & $<0.001$ \\
\hline Antiplatelet user, n (\%) & $450(52.4)$ & $6309(49.0)$ & $5453(50.3)$ & $1235(50.6)$ & $135(48.7)$ & 0.112 \\
\hline Statin user, n (\%) & $338(39.4)$ & $5192(40.3)$ & $4407(40.7)$ & $978(40.1)$ & III(39.8) & 0.944 \\
\hline Insulin user, n (\%) & $518(60.4)$ & $7984(61.9)$ & $6647(61.2)$ & $1434(58.5)^{\mathrm{a}}$ & $164(58.8)$ & 0.028 \\
\hline Metformin user, n (\%) & $162(18.9)^{\mathrm{a}}$ & $2972(23.0)$ & $27 I I(25.0)^{\mathrm{a}}$ & $593(24.2)$ & $70(25.1)$ & $<0.001$ \\
\hline FBG $(\mathrm{mmol} / \mathrm{L})$ & $10.4 \pm 5.8$ & $10.2 \pm 5.3$ & $10.1 \pm 4.9$ & $10.2 \pm 4.7$ & $10.2 \pm 5.4$ & 0.125 \\
\hline HbAlc (\%) & $8.3 \pm 2.4$ & $8.2 \pm 2.2$ & $8.1 \pm 2.0^{a}$ & $8.1 \pm 2.0$ & $8.1 \pm 2.0$ & 0.010 \\
\hline Hypoglycemia, n (\%) & $17(1.8)$ & 179(1.3) & $128(1.1)$ & $19(0.7)^{\mathrm{a}}$ & $3(1.0)$ & 0.032 \\
\hline $\mathrm{T}-\mathrm{CHO}(\mathrm{mmol} / \mathrm{L})$ & $4.3 \pm 1.2^{\mathrm{a}}$ & $4.5 \pm 1.4$ & $4.5 \pm 1.3^{\mathrm{a}}$ & $4.6 \pm 1.3^{\mathrm{a}}$ & $4.6 \pm 1.2$ & $<0.001$ \\
\hline HDL-C (mmol/L) & $1.2 \pm 0.3^{\mathrm{a}}$ & $1.1 \pm 0.3$ & $1.1 \pm 0.3^{\mathrm{a}}$ & $1.1 \pm 0.3^{\mathrm{a}}$ & $1.1 \pm 0.3$ & $<0.001$ \\
\hline LDL-C (mmol/L) & $2.6 \pm 0.9^{\mathrm{a}}$ & $2.7 \pm 0.9$ & $2.8 \pm 1.0^{\mathrm{a}}$ & $2.8 \pm 0.9^{\mathrm{a}}$ & $2.8 \pm 1.0$ & $<0.001$ \\
\hline Triglyceride (mmol/L) & $1.2(0.8-1.7)^{\mathrm{a}}$ & $1.4(1.0-2.1)$ & $1.6(1.1-2.3)^{a}$ & $1.7(1.2-2.5)^{\mathrm{a}}$ & $1.8(1.3-2.5)^{\mathrm{a}}$ & $<0.001$ \\
\hline Apolipoprotein-AI (g/L) & $1.1 \pm 0.3$ & $1.1 \pm 0.3$ & $1.1 \pm 0.3^{\mathrm{a}}$ & $1.1 \pm 0.3^{\mathrm{a}}$ & $1.1 \pm 0.3$ & 0.023 \\
\hline Apolipoprotein-B (g/L) & $0.8 \pm 0.2^{\mathrm{a}}$ & $0.8 \pm 0.2$ & $0.8 \pm 0.3^{\mathrm{a}}$ & $0.8 \pm 0.2^{\mathrm{a}}$ & $0.8 \pm 0.3$ & $<0.001$ \\
\hline Lipoprotein-a (mg/L) & $232(117.5-400.5)^{\mathrm{a}}$ & $197(103-349)$ & $186(97-330.5)^{\mathrm{a}}$ & $182(99-326)^{a}$ & $160(73.8-3 \mid 8.3)^{\mathrm{a}}$ & $<0.001$ \\
\hline BUN (mmol/L) & $7.3 \pm 5.1^{a}$ & $6.8 \pm 4.6$ & $6.7 \pm 4.5$ & $6.7 \pm 4.6$ & $6.8 \pm 4.6$ & 0.003 \\
\hline $\mathrm{SCr}$ (umol/L) & $73(60-95)$ & $74(61-92)$ & $76(63-94)^{\mathrm{a}}$ & $75(62-94)^{a}$ & $74(60-92.5)$ & $<0.001$ \\
\hline SUA (umol/L) & $272.2 \pm 131.7^{\mathrm{a}}$ & $289.3 \pm|2| .3$ & $305.3 \pm 123.2^{\mathrm{a}}$ & $313.1 \pm 133.2^{\mathrm{a}}$ & $334.4 \pm 143.8^{\mathrm{a}}$ & $<0.001$ \\
\hline
\end{tabular}

Notes: Data are mean $\pm S D$, median (interquartile range), or $n(\%) .{ }^{a} \mathrm{P}<0.05$ compared to Normal weight group.

Abbreviations: BMI, body mass index; CHD, coronary heart disease; FBG, fasting blood-glucose; HbAlc, glycosylated hemoglobin; T-CHO, total cholesterol; HDL-C, highdensity lipoprotein cholesterol; LDL-C, low-density lipoprotein cholesterol; BUN, blood urea nitrogen; SCr, serum creatinine; SUA, serum uric acid.

both ends of the curve. In patients without hypertension, BMI and CHD risk showed a dose-dependent relationship, with the highest risk found in the severe obesity group (OR 2.51, 95\% CI, 0.85-7.42).

\section{Interaction of Cardiovascular Risk Factor BMI and Hypertension in Coronary Heart Disease}

Figure 3 shows the independent effect and additive interaction of BMI and hypertension on CHD. Patients with BMI $18.5-24.9 \mathrm{~kg} / \mathrm{m}^{2}$ without hypertension were taken as controls $(\mathrm{OR}=1)$. The $\mathrm{OR}$ value of hypertension alone on CHD was increased from 1.29 (95\% CI 1.06-1.56) in BMI $30.0-34.9 \mathrm{~kg} / \mathrm{m}^{2}$ group to 1.35 (95\% CI 1.11-1.62) in BMI $\leq 18.5 \mathrm{~kg} / \mathrm{m}^{2}$ group, and an additive interaction was found between hypertension and body weight in CHD risk, especially in the low weight group (RERI:2.2, 95\% CI 0.9 3.5). A multiplicative interaction was also observed between the two risk factors, OR 2.62 (95\% CI 1.743.93) $(\mathrm{P}<0.01)$. After the additive interaction was considered, BMI and CHD risk showed a smile curve relationship. The risk of CHD was the highest in the low weight group with OR 4.23 (95\% CI 3.34-5.35) and severe obesity group with OR 4.15 (95\% CI 2.97-5.81) at both ends of BMI.

Table 3 shows the statistical analysis of the additive interaction to support the results. The contribution of the additive interaction varied for different BMI groups, with the attributable proportion (AP) significantly higher in the low weight group than in the severe obesity group, 0.52 (95\% CI $0.35-0.69)$ vs 0.18 (95\% CI -0.59 to 0.95$)$. The AP was -0.07 (95\% CI -0.21 to 0.08$)$ in the overweight group and $0.03(95 \% \mathrm{CI}-0.24$ to 0.31$)$ in mild obesity. This result indicates that in low weight patients with cooccurring diabetes and hypertension, $52 \%$ of CHD risk can be attributed to the additive interaction or combination between weight and hypertension. 


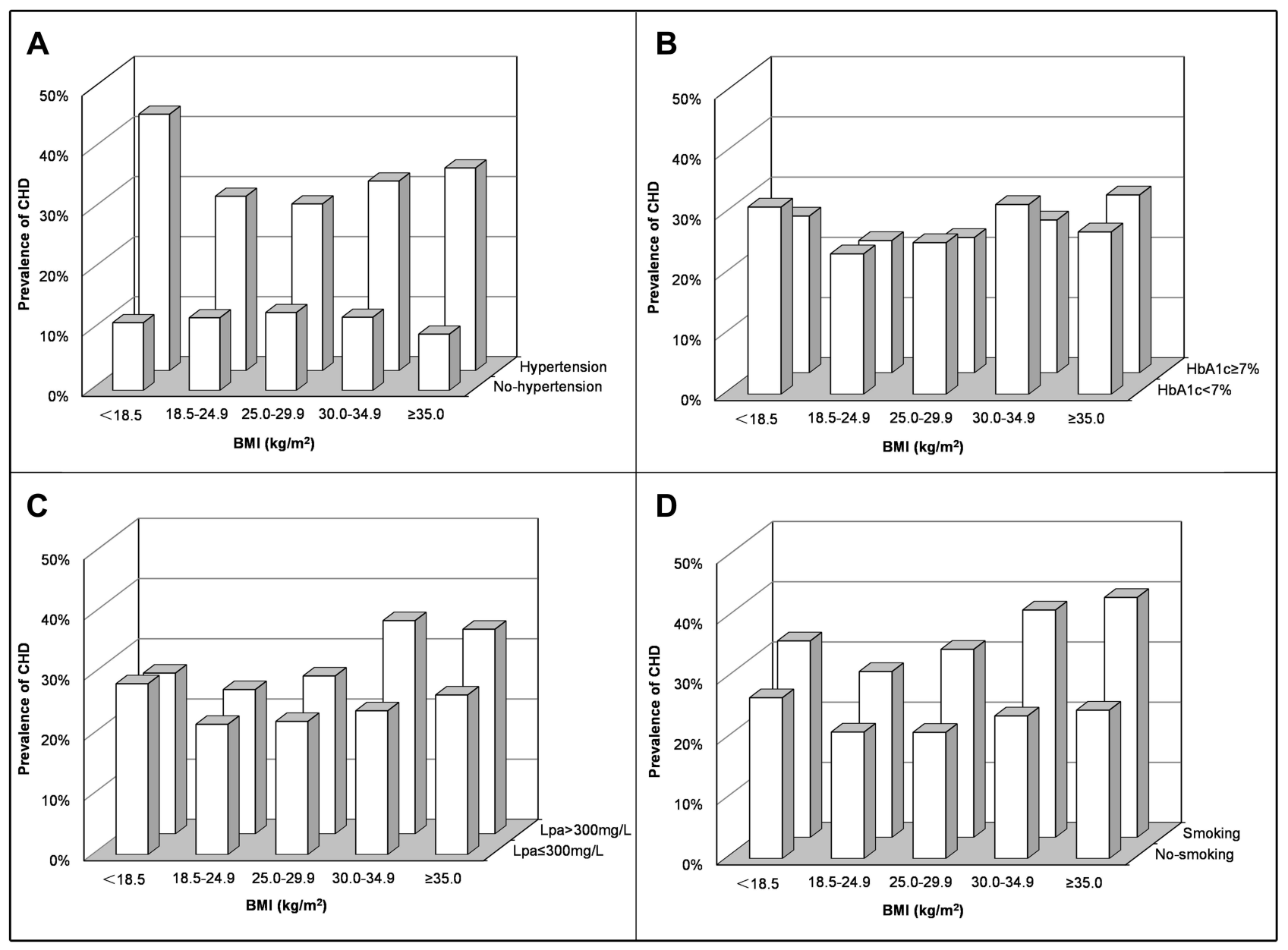

Figure 2 Prevalence of coronary heart disease in patients with type 2 diabetes by cardiovascular risk factors and body mass index levels. Patients were classified according to the history of hypertension (A), HbAlc (B), Lpa (C) and smoking (D).

Abbreviations: CHD, coronary heart disease; BMI, body mass index; HbAIc, glycosylated hemoglobin; Lpa, lipoprotein-a.

\section{Discussion}

The role of cardiovascular risk factors and their interactions in CHD risk was evaluated in patients with type 2 diabetes. Hypertension was found to be the most influential cardiovascular risk factor for CHD across the BMI groups and had similar effects at all BMI levels. An additive interaction was observed between hypertension and body weight on CHD risk, especially in the low weight population. BMI and CHD risk showed a smile curve after additive interaction was considered. In the low weight patients with co-occurring diabetes and hypertension, $52 \%$ of the CHD risk can be attributed to this additive interaction.

CHD is the most common cause of death in patients with diabetes. Stratification according to different risk factors and CHD screening with different intensities must be conducted for people with different risk levels for the accurate use of limited medical resources. Several clinical guidelines have recommended strict control of body weight, which is a well-known risk factor for CHD, for CHD prevention. ${ }^{19}$ However, the role of body weight in CHD is still controversial. CHD risk gradually increases with the increase in BMI. ${ }^{20,21}$ However, most studies have only focused on the obese population, and the low body weight population is rarely involved. The present work indicates that in patients with diabetes and hypertension, the risk of CHD in the low weight group was higher than that in the normal weight group and even higher than in the overweight group. This finding is consistent with other clinical studies that focused on low body weight. A large cohort study found ${ }^{22}$ a reduced risk of cardiovascular death in overweight and moderately obese patients and an increased risk of cardiovascular death in underweight patients. A study of 54,285 patients with 4-year follow-up 
Table 2 Incidence Rates and ORs for CHD According to BMI by History of HP in Patients with Type 2 Diabetes

\begin{tabular}{|c|c|c|c|c|c|}
\hline & $\begin{array}{l}\text { Low Weight } \\
\left(<18.5 \mathrm{~kg} / \mathrm{m}^{2}\right)\end{array}$ & $\begin{array}{l}\text { Normal Weight } \\
\left(18.5-24.9 \mathrm{~kg} / \mathrm{m}^{2}\right)\end{array}$ & $\begin{array}{l}\text { Overweight } \\
\left(25.0-29.9 \mathrm{~kg} / \mathrm{m}^{2}\right)\end{array}$ & $\begin{array}{l}\text { Mild Obesity } \\
\left(30.0-34.9 \mathrm{~kg} / \mathrm{m}^{2}\right)\end{array}$ & $\begin{array}{l}\text { Severe Obesity } \\
\left(\geq 35.0 \mathrm{~kg} / \mathrm{m}^{2}\right)\end{array}$ \\
\hline \multicolumn{6}{|c|}{ Prevalence, $n$ (\%) } \\
\hline HP & $216(42.7)$ & $2550(29.0)$ & $2382(27.8)$ & $685(31.6)$ & $83(33.7)$ \\
\hline No-HP & $52(11.3)$ & $685(12.1)$ & $470(13.0)$ & $73(12.2)$ & $6(9.4)$ \\
\hline \multicolumn{6}{|c|}{ OR $(95 \% \mathrm{Cl})$} \\
\hline \multicolumn{6}{|c|}{ Basic regression model ${ }^{\mathrm{a}}$} \\
\hline HP & $1.67(1.37-2.03)$ & I.00 (Ref) & $1.02(0.95-1.09)$ & $1.28(1.14-1.42)$ & $1.68(1.26-2.25)$ \\
\hline No-HP & $0.74(0.54-1.01)$ & I.00 (Ref)* & $1.25(1.1-1.43)$ & I.54(I.I7-2.03) & $1.84(0.74-4.55)$ \\
\hline \multicolumn{6}{|c|}{ Main regression model ${ }^{b}$} \\
\hline HP & $1.78(1.43-2.23)$ & I.00 (Ref) & $1.05(0.96-1.13)$ & $1.27(1.12-1.44)$ & $1.72(1.24-2.39)$ \\
\hline No-HP & $0.62(0.44-0.88)$ & 1.00 (Ref)* & $1.30(|| 2-.|.5|)$ & $1.69(1.23-2.31)$ & $2.5 \mathrm{I}(0.85-7.42)$ \\
\hline
\end{tabular}

Notes: äasic models include adjustment for sex, age, and diabetes duration. 'bain models additionally include smoking, drinking, use of antiplatelet, statin, insulin, and metformin, FBG, HbAlc, hypoglycemia, total cholesterol, LDL-C, triglyceride, lipoprotein-a, SCr, and SUA; *The same model as for HP group except patients without hypertension and BMI $18.5-24.9 \mathrm{~kg} / \mathrm{m}^{2}$ constitute the reference group.

Abbreviations: CHD, coronary heart disease; BMl, body mass index; HP, hypertension; Ref, reference group; FBG, fasting blood-glucose; HbAIc, glycosylated hemoglobin; LDL-C, low-density lipoprotein cholesterol; SCr, serum creatinine; SUA, serum uric acid.

showed ${ }^{23}$ that underweight patients $\left(\mathrm{BMI}<18 \mathrm{~kg} / \mathrm{m}^{2}\right)$ have the highest risk of all cardiovascular events. The results from National Health and Nutrition Examination Survey (NHANES) showed that cardiovascular disease mortality risk was higher in underweight people. ${ }^{24}$ Therefore, interventions for cardiovascular risk factors should focus not only on obese people, but also on people with low weight. Avoiding a low body weight is more likely to be neglected

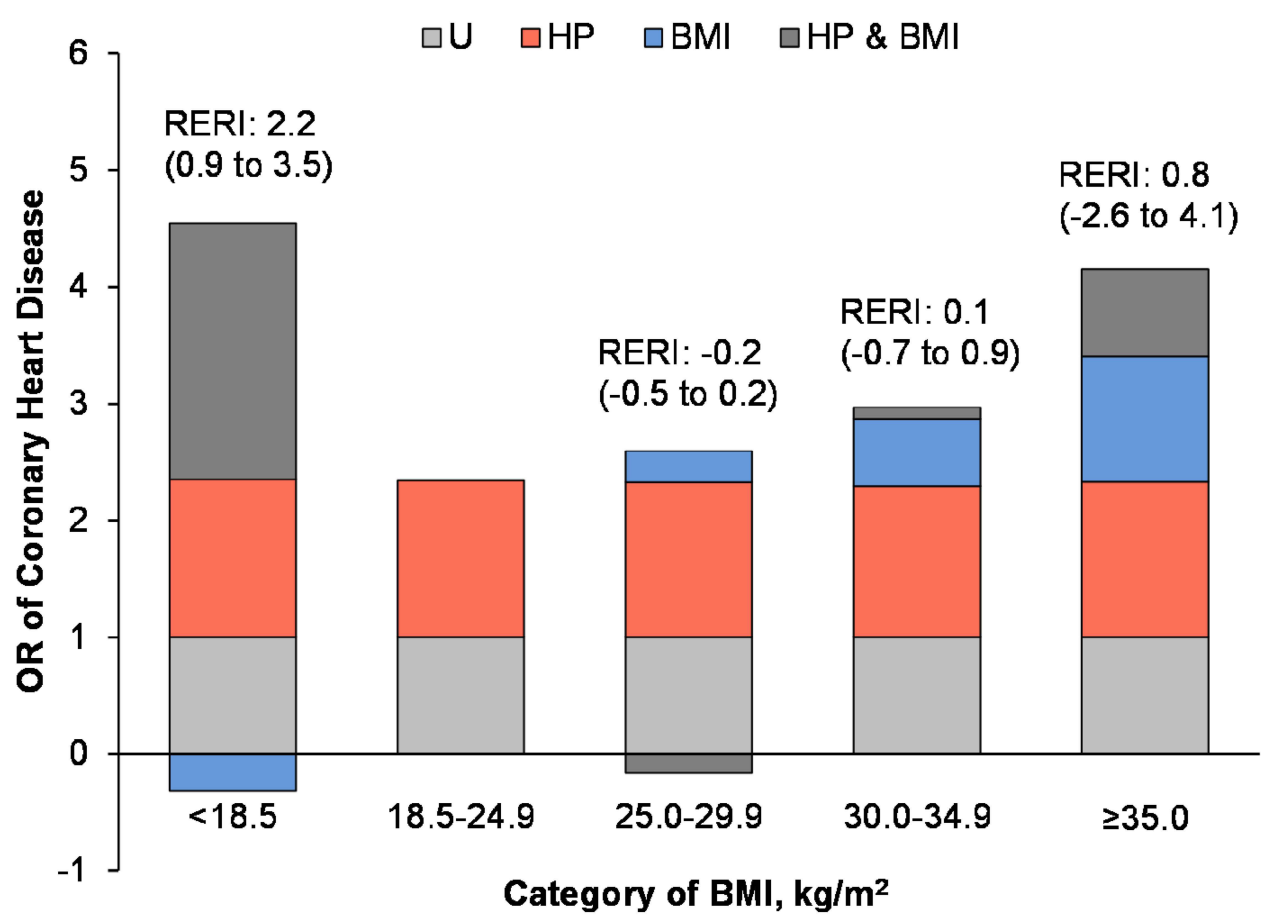

Figure 3 Additive interaction of BMI and hypertension on risk of CHD by BMI presented as odds ratio partitioned into relative excess risks due to BMI, hypertension, and their interaction.

Notes: All ORs are adjusted for sex, age, diabetes duration, smoking, drinking, use of antiplatelet, statin, insulin, and metformin, FBG, HbAIc, hypoglycemia, total cholesterol, LDL-C, triglyceride, lipoprotein-a, SCr, and SUA.

Abbreviations: BMI, body mass index; $\mathrm{CHD}$, coronary heart disease; HP, hypertension; $\mathrm{U}, \mathrm{BMI} 18.5$ to $<25.0 \mathrm{~kg} / \mathrm{m}^{2}$ and without hypertension; RERI, relative excess risk due to interaction; FBG, fasting blood-glucose; HbAlc, glycosylated hemoglobin; LDL-C, low-density lipoprotein cholesterol; SCr, serum creatinine; SUA, serum uric acid. 
Table 3 Statistics for Additive Interaction Between BMI and HP in Multivariable-Adjusted Models of Incident CHD in Patients with Type 2 Diabetes Mellitus

\begin{tabular}{|c|c|c|c|c|c|}
\hline \multirow[b]{2}{*}{ Additive Interaction Statistic } & \multicolumn{5}{|c|}{ BMI Category $\left(\mathrm{kg} / \mathrm{m}^{2}\right)$} \\
\hline & $\begin{array}{c}\text { Low Weight } \\
(<18.5)\end{array}$ & $\begin{array}{c}\text { Normal Weight } \\
\quad(18.5-24.9)\end{array}$ & $\begin{array}{l}\text { Overweight } \\
(25.0-29.9)\end{array}$ & $\begin{array}{l}\text { Mild Obesity } \\
(30.0-34.9)\end{array}$ & $\begin{array}{c}\text { Severe Obesity } \\
(\geq 35.0)\end{array}$ \\
\hline RERI (95\% Cl) & $2.19(0.88,3.50)$ & Reference* & $-0.16(-0.52,0.20)$ & $0.10(-0.71,0.91)$ & $0.75(-2.64,4.14)$ \\
\hline Attributable proportion ${ }^{\mathrm{a}}(95 \% \mathrm{Cl})$ & $0.52(0.35,0.69)$ & Reference* & $-0.07(-0.21,0.08)$ & $0.03(-0.24,0.31)$ & $0.18(-0.59,0.95)$ \\
\hline Synergy index $(95 \% \mathrm{Cl})$ & $3.1 \mathrm{I}(\mathrm{I} .75,5.53)$ & Reference* & $0.90(0.72, \mathrm{I} .12)$ & $1.05(0.69,1.62)$ & $1.3 \mathrm{I}(0.35,4.89)$ \\
\hline
\end{tabular}

Notes: *The reference group is patients with BMI $18.5-24.9 \mathrm{~kg} / \mathrm{m}^{2}$ and without HP. ${ }^{\text {Th }}$ The proportion of CHD risk that is attributable to the additive interaction between BMI and hypertension.

Abbreviations: BMI, body mass index; CHD, coronary heart disease; HP, hypertension; RERI, relative excess risk due to interaction.

than weight loss in obese patients. This phenomenon is called the obesity paradox ${ }^{25}$ and has multiple explanations, such as sarcopenic obesity, the limitation of BMI due to the inability to distinguish fat from skeletal muscle weight, ${ }^{26}$ and selection bias. ${ }^{27} \mathrm{We}$ found that persons with low BMI had lower LDL-C level and were older than those with normal BMI, it may be due to the longterm strict control of blood lipid, which prolongs the life span of patients, leading to a longer course of chronic diseases such as diabetes. The results served as a reminder that for the clinical prevention and control of CHD, the focus should not just be the overweight and obese people but also low weight people. The reasons for the increased risk of CHD should be clarified, and individualized intervention plans should be formulated.

In this study, patients with different BMI levels were further classified according to the presence or absence of hypertension. The relationship between BMI and CHD risk was exhibited a smile curve in patients with hypertension and dose dependency in the population without hypertension. This finding suggests that the contribution of BMI to CHD is influenced by hypertension. The two risk factors are not independent but have an interactive effect. This view is consistent with some recent findings. Recent studies ${ }^{28-31}$ showed that the risk of cardiovascular disease is dependent on obesity and the presence of other metabolic disorders. The risk of cardiovascular disease is lower in metabolically healthy obese people than in metabolically unhealthy obese people. The risk of cardiovascular disease in metabolically unhealthy normal weight individuals is similar to that in metabolically unhealthy obese individuals. The present study found that the risk of CHD is greatly increased in low weight patients with hypertension, with the synergistic contribution of the two factors reaching $52 \%$. This additive interaction seriously affects diseases. A long-term follow- up study ${ }^{11}$ showed that the interaction between BMI and hypertension contributes $36 \%$ to diabetes in people with a BMI of $30.0-34.9 \mathrm{~kg} / \mathrm{m}^{2}$. The current results provided a new interpretation of the phenomenon of obesity paradox. In addition to the above reasons, the interactive effects play a huge role in this phenomenon. Most epidemic scholars believe that multiply interactions reflect statistical interaction, and evaluation on biological interactions should be based on additive interactions. ${ }^{32}$ Hence, the additive effect was the focus of our research. Although the interaction between hypertension and low body weight is only based on observational data, previous researches have provided some biological basis for this finding. Studies have shown that the uptake of norepinephrine in neurons was severely impaired in patients with lean hypertension, but not at all in obese hypertensive patients. ${ }^{33}$ The consequences of neuronal norepinephrine reuptake disorder are the promotion of norepinephrine overflow from sympathetic nerves to plasma, and lead to overstimulation in cardiac sympathetic nerve. ${ }^{34}$ As is known that the ongoing activation of sympathetic nervous system is causally associated with atherosclerosis progression $^{35}$ and endothelial dysfunction. ${ }^{36}$

To avoid the interaction between hypertension and low body weight, hypoglycemic drugs with weight loss effects should be used more carefully in diabetic patients with hypertension and low BMI. GLP-1 agonists and SGLT-2 inhibitors are listed in the international guidelines as firstline medications for T2DM patients with high cardiovascular risk because the cardiovascular benefits, ${ }^{37,38}$ while both are also associated with weight loss. It is worth considering that if patients loss weight excessively to underweight after taking GLP-1 agonists or SGLT-2 inhibitors, low body weight may cancel out the cardiovascular benefits of the medication. Therefore, we suggest that for patients using GLP-1 agonists or SGLT-2 inhibitors, body 
weight should be monitored regularly, and if significant weight loss or underweight is found, treatment should be adjusted in time. Weight status should be an important consideration when choosing treatment.

This study has certain limitations. First, although the studies involved a large population, no further follow-up data have been collected. Hence, the causal explanation of conclusion may exhibit deviation. Further cohort study is needed to verify the results. Second, the study population was patients with diabetes in a university hospital. The severity and type of disease, the measures of clinical intervention, and other aspects of this population may differ from those of the general diabetic population. Therefore, the results of this study may not be applicable to the general population. A broad multicenter study must be conducted in the future. Third, the weight data only included BMI. Indices such as waist-to-hip ratio, visceral fat evaluation, and BMI change were not considered. This aspect should be further improved in subsequent studies.

\section{Conclusion}

In patients with diabetes, the risk of CHD markedly increased by the interaction of low body weight and hypertension. Compared with patients without hypertension, avoiding a low body weight seems to be especially important with hypertension. Therefore, weight intervention in patients with low body weight may provide great clinical benefits.

\section{Data Sharing Statement}

The data that support the findings of this study are available from the corresponding author upon reasonable request.

\section{Ethics Approval and Consent to Participate}

This study was approved by the Research Ethics Committee of the Affiliated ZhongDa Hospital of Southeast University (Approved No. of ethic committee: 2020ZDSYLL028-P01). Due to the retrospective nature of the study and all identifying data of the patients could not be distinguished, the consent of the patients to participate was waived. The study was carried out in conformity to the Declaration of Helsinki.

\section{Acknowledgments}

We would like to express our heartfelt thanks to all the participants in this study. This work was supported by the National Natural Science Foundation of China (No. 81570732, Shaohua Wang; and No. 81870568, Shaohua Wang).

\section{Author Contributions}

All authors made substantial contributions to conception and design, acquisition of data, or analysis and interpretation of data, took part in drafting the article or revising it critically for important intellectual content, agreed to submit to the current journal, gave final approval to the version to be published, and agree to be accountable for all aspects of the work.

\section{Disclosure}

The authors report no conflicts of interest in this work.

\section{References}

1. Haffner S, Lehto S, Rönnemaa T, Pyörälä K, Laakso M. Mortality from coronary heart disease in subjects with type 2 diabetes and in nondiabetic subjects with and without prior myocardial infarction. $N$ Engl J Med. 1998;339(4):229-234. doi:10.1056/nejm199807233390404

2. Expert Panel on Detection E, and Treatment of High Blood Cholesterol in Adults. Executive summary of the third report of the National Cholesterol Education Program (NCEP) expert panel on detection, evaluation, and treatment of high blood cholesterol in adults (adult treatment panel III). JAMA. 2001;285(19):2486-2497. doi:10.1001/jama.285.19.2486.

3. Ali Raza J, Movahed A. Current concepts of cardiovascular diseases in diabetes mellitus. Int $J$ Cardiol. 2003;89:123-134. doi:10.1016/ s0167-5273(02)00510-7

4. Lee J, Ma S, Heng D, Chew S, Hughes K, Tai E. Hypertension, concurrent cardiovascular risk factors and mortality: the Singapore Cardiovascular Cohort Study. J Hum Hypertens. 2008;22 (7):468-474. doi:10.1038/jhh.2008.16

5. Chen G, McAlister F, Walker R, Hemmelgarn B, Campbell N. Cardiovascular outcomes in Framingham participants with diabetes: the importance of blood pressure. Hypertension. 2011;57(5):891-897. doi:10.1161/hypertensionaha.110.162446

6. Lehto S, Rönnemaa T, Pyörälä K, Laakso M. Cardiovascular risk factors clustering with endogenous hyperinsulinaemia predict death from coronary heart disease in patients with type II diabetes. Diabetologia. 2000;43(2):148-155. doi:10.1007/s001250050023

7. Chung W, Erion K, Florez J, et al. Precision medicine in diabetes: a consensus report from the American Diabetes Association (ADA) and the European Association for the Study of Diabetes (EASD). Diabetes Care. 2020;43(7):1617-1635. doi:10.2337/dci20-0022

8. Patel A, MacMahon S, Chalmers J, et al. Intensive blood glucose control and vascular outcomes in patients with type 2 diabetes. $N$ Engl J Med. 2008;358(24):2560-2572. doi:10.1056/NEJMoa0802987

9. Gerstein H, Miller M, Byington R, et al. Effects of intensive glucose lowering in type 2 diabetes. $N$ Engl J Med. 2008;358(24):2545-2559. doi:10.1056/NEJMoa0802743

10. Zhao D, Liu J, Xie W, Qi Y. Cardiovascular risk assessment: a global perspective. Nat Rev Cardiol. 2015;12(5):301-311. doi:10.1038/ nrcardio. 2015.28

11. Timpka S, Stuart J, Tanz L, Hu F, Franks P, Rich-Edwards J. Postpregnancy BMI in the progression from hypertensive disorders of pregnancy to type 2 diabetes. Diabetes Care. 2019;42(1):44-49. doi:10.2337/dc18-1532

12. Timpka S, Stuart J, Tanz L, Rimm E, Franks P, Rich-Edwards J. Lifestyle in progression from hypertensive disorders of pregnancy to chronic hypertension in Nurses' Health Study II: observational cohort study. BMJ. 2017;358:j3024. doi:10.1136/bmj.j3024 
13. Feldstein C, Julius S. The complex interaction between overweight, hypertension, and sympathetic overactivity. J Am Soc Hypertens. 2009;3(6):353-365. doi:10.1016/j.jash.2009.10.001

14. Musameh M, Wang W, Nelson CP, et al. Analysis of gene-gene interactions among common variants in candidate cardiovascular genes in coronary artery disease. PLoS One. 2015;10(2):e0117684. doi:10.1371/journal.pone.0117684

15. Napoli C, Benincasa G, Schiano C, Salvatore M. Differential epigenetic factors in the prediction of cardiovascular risk in diabetic patients. Eur Heart J Cardiovasc Pharmacother. 2020;6 (4):239-247. doi:10.1093/ehjcvp/pvz062

16. Song Y, Shan Z, Liu X, et al. An updated meta-analysis showed smoking modify the association of GSTM1 null genotype on the risk of coronary heart disease. Biosci Rep. 2021;41(2). doi:10.1042/ bsr20200490

17. Wang C, Lê-Scherban F, Taylor J, et al. Associations of job strain, stressful life events, and social strain with coronary heart disease in the Women's Health initiative observational study. J Am Heart Assoc. 2021;10(5):e017780. doi:10.1161/jaha.120.017780

18. Andersson T, Alfredsson L, Källberg H, Zdravkovic S, Ahlbom A. Calculating measures of biological interaction. Eur J Epidemiol. 2005;20(7):575-579. doi:10.1007/s10654-005-7835-x

19. Kivimäki M, Kuosma E, Ferrie J, et al. Overweight, obesity, and risk of cardiometabolic multimorbidity: pooled analysis of individuallevel data for 120813 adults from 16 cohort studies from the USA and Europe. Lancet Public Health. 2017;2(6):e277-e285. doi:10.1016/s2468-2667(17)30074-9

20. Dale C, Fatemifar G, Palmer T, et al. Causal associations of adiposity and body fat distribution with coronary heart disease, stroke subtypes, and type 2 diabetes mellitus: a Mendelian randomization analysis. Circulation. 2017;135(24):2373-2388. doi:10.1161/ circulationaha.116.026560

21. Larsson S, Bäck M, Rees J, Mason A, Burgess S. Body mass index and body composition in relation to 14 cardiovascular conditions in UK Biobank: a Mendelian randomization study. Eur Heart J. 2020;41(2):221-226. doi:10.1093/eurheartj/ehz388

22. Doehner W, Gerstein H, Ried J, et al. Obesity and weight loss are inversely related to mortality and cardiovascular outcome in prediabetes and type 2 diabetes: data from the ORIGIN trial. Eur Heart J. 2020;41(28):2668-2677. doi:10.1093/eurheartj/ehaa293

23. Hansel B, Roussel R, Elbez Y, et al. Cardiovascular risk in relation to body mass index and use of evidence-based preventive medications in patients with or at risk of atherothrombosis. Eur Heart J. 2015;36 (40):2716-2728. doi:10.1093/eurheartj/ehv347

24. Luijckx E, Lohse T, Faeh D, Rohrmann S. Joints effects of BMI and smoking on mortality of all-causes, CVD, and cancer. Cancer Causes Control. 2019;30(5):549-557. doi:10.1007/s10552-019-01160-8

25. Doehner W. Critical appraisal of the obesity paradox in cardiovascular disease: how to manage patients with overweight in heart failure? Heart Fail Rev. 2014;19(5):637-644. doi:10.1007/s10741-014-9425-z
26. Lavie C, McAuley P, Church T, Milani R, Blair S. Obesity and cardiovascular diseases: implications regarding fitness, fatness, and severity in the obesity paradox. $J$ Am Coll Cardiol. 2014;63 (14):1345-1354. doi:10.1016/j.jacc.2014.01.022

27. Elagizi A, Kachur S, Lavie C, et al. An overview and update on obesity and the obesity paradox in cardiovascular diseases. Prog Cardiovasc Dis. 2018;61(2):142-150. doi:10.1016/j.pcad.20 18.07.003

28. Eckel N, Meidtner K, Kalle-Uhlmann T, Stefan N, Schulze M. Metabolically healthy obesity and cardiovascular events: a systematic review and meta-analysis. Eur J Prev Cardiol. 2016;23 (9):956-966. doi:10.1177/2047487315623884

29. Kramer C, Zinman B, Retnakaran R. Are metabolically healthy overweight and obesity benign conditions?: A systematic review and meta-analysis. Ann Intern Med. 2013;159(11):758-769. doi:10.7326/0003-4819-159-11-201312030-00008

30. Lassale C, Tzoulaki I, Moons K, et al. Separate and combined associations of obesity and metabolic health with coronary heart disease: a pan-European case-cohort analysis. Eur Heart J. 2018;39 (5):397-406. doi:10.1093/eurheartj/ehx448

31. Caleyachetty R, Thomas G, Toulis K, et al. Metabolically healthy obese and incident cardiovascular disease events among 3.5 million men and women. J Am Coll Cardiol. 2017;70(12):1429-1437. doi:10.1016/j.jacc.2017.07.763

32. Rothman KJ, Greenland S, Lash TL. Modern Epidemiology. 3rd ed. Philadelphia: Wolters KIuwer, Lippincott Williams and Wilhns; 2008.

33. Rumantir M, Kaye D, Jennings G, Vaz M, Hastings J, Esler M. Phenotypic evidence of faulty neuronal norepinephrine reuptake in essential hypertension. Hypertension. 2000;36(5):824-829. doi:10.1161/01.hyp.36.5.824

34. Esler M, Wallin G, Dorward P, et al. Effects of desipramine on sympathetic nerve firing and norepinephrine spillover to plasma in humans. Am J Physiol. 1991;260:R817-R823. doi:10.1152/ ajpregu.1991.260.4.R817

35. Lichtor T, Davis H, Johns L, Vesselinovitch D, Wissler R, Mullan S. The sympathetic nervous system and atherosclerosis. J Neurosurg. 1987;67(6):906-914. doi:10.3171/jns.1987.67.6.0906

36. Amiya E, Watanabe M, Komuro I. The Relationship between Vascular Function and the Autonomic Nervous System. Ann Vasc Dis. 2014;7(2):109-119. doi:10.3400/avd.ra.14-00048

37. Vesa C, Popa L, Popa A, et al. Current data regarding the relationship between type 2 diabetes mellitus and cardiovascular risk factors. Diagnostics. 2020;10(5):314. doi:10.3390/diagnostics10050314

38. Iorga RA, Bacalbasa N, Carsote M, et al. Metabolic and cardiovascular benefits of GLP-1 agonists, besides the hypoglycemic effect. Exp Ther Med. 2020;20(3):2396-2400. doi:10.3892/etm.2020.8714

Diabetes, Metabolic Syndrome and Obesity: Targets and Therapy

\section{Publish your work in this journal}

Diabetes, Metabolic Syndrome and Obesity: Targets and Therapy is an international, peer-reviewed open-access journal committed to the rapid publication of the latest laboratory and clinical findings in the fields of diabetes, metabolic syndrome and obesity research. Original research, review, case reports, hypothesis formation, expert opinion and commentaries are all considered for publication. The manuscript management system is completely online and includes a very quick and fair peer-review system, which is all easy to use. Visit http://www.dovepress.com/testimonials.php to read real quotes from published authors. 